\title{
Cadmium-Zinc-Telluride Myocardial Perfusion Imaging in Obese Patients
}

\author{
Michael Fiechter*1,2, Cathérine Gebhard*1, Tobias A. Fuchs ${ }^{1}$, Jelena R. Ghadri ${ }^{1}$, Julia Stehli ${ }^{1}$, Egle Kazakauskaite ${ }^{1}$, \\ Bernhard A. Herzog ${ }^{1}$, Aju P. Pazhenkottil ${ }^{1}$, Oliver Gaemperli ${ }^{1}$, and Philipp A. Kaufmann ${ }^{1,2}$ \\ ${ }^{1}$ Department of Radiology, Cardiac Imaging, University Hospital Zurich, Zurich, Switzerland; and ${ }^{2}$ Zurich Center for Integrative \\ Human Physiology (ZIHP), University of Zurich, Zurich, Switzerland
}

\begin{abstract}
We have evaluated the impact of increased body mass on the quality of myocardial perfusion imaging using a latest-generation $\gamma$-camera with cadmium-zinc-telluride semiconductor detectors in patients with high $\left(\geq 40 \mathrm{~kg} / \mathrm{m}^{2}\right)$ or very high $(\geq 45$ $\mathrm{kg} / \mathrm{m}^{2}$ ) body mass index (BMI). Methods: We enrolled 81 patients, including 18 with no obesity (BMI $\left.<30 \mathrm{~kg} / \mathrm{m}^{2}\right), 17$ in World Health Organization obese class I (BMI, 30-34.9 kg/m²), 15 in class II (BMI, 35-39.9 kg/m²), and 31 in class III (BMI $\geq 40$ $\mathrm{kg} / \mathrm{m}^{2}$ ), including 15 with $\mathrm{BMI} \geq 45 \mathrm{~kg} / \mathrm{m}^{2}$. Image quality was scored as poor (1), moderate (2), good (3), or excellent (4). Patients with $\mathrm{BMI} \geq 45 \mathrm{~kg} / \mathrm{m}^{2}$ and nondiagnostic image quality $(\leq 2)$ were rescanned after repositioning to better center the heart in the field of view. Receiver-operating-curve analysis was applied to determine the BMI cutoff required to obtain diagnostic image quality ( $\geq 3$ ). Results: Receiver-operating-curve analysis resulted in a cutoff BMl of $39 \mathrm{~kg} / \mathrm{m}^{2}(P<0.001)$ for diagnostic image quality. In patients with $\mathrm{BMI} \geq 40 \mathrm{~kg} / \mathrm{m}^{2}$, image quality was nondiagnostic in $81 \%$; after CT-based attenuation correction this decreased to $55 \%$. Repositioning further improved image quality. Rescanning on a conventional SPECT camera resulted in diagnostic image quality in all patients with $\mathrm{BMI} \geq 45 \mathrm{~kg} / \mathrm{m}^{2}$. Conclusion: Patients with BMI $\geq 40 \mathrm{~kg} / \mathrm{m}^{2}$ should be scheduled for myocardial perfusion imaging on a conventional SPECT camera, as it is difficult to obtain diagnostic image quality on a cadmium-zinc-telluride camera.
\end{abstract}

Key Words: BMI; CZT; chest wall circumference; obesity; SPECT

J Nucl Med 2012; 53:1401-1406

DOI: 10.2967/jnumed.111.102434

\section{A}

mong the many refinements of SPECT myocardial perfusion imaging (MPI) that have occurred over the past decade, most have been related to new reconstruction algorithms ( 1 , early imaging protocols $(2,3)$, and tracer development rather than to fundamental advancements of hardware. Only recently has a new generation of cardiac $\gamma$-cameras emerged using the

\footnotetext{
Received Dec. 27, 2011; revision accepted Apr. 2, 2012.

For correspondence or reprints contact: Philipp A. Kaufmann, University Hospital Zurich, Ramistrasse 100, NUK C 42, CH-8091 Zurich, Switzerland.

E-mail: pak@usz.ch

Published online Jun. 29, 2012.

${ }^{*}$ Contributed equally to this work

COPYRIGHT (C) 2012 by the Society of Nuclear Medicine and Molecular Imaging, Inc.
}

concept of miniaturized cadmium-zinc-telluride (CZT) semiconductor detectors serially aligned around the patient. The multipinhole design renders rotation of the camera around the patient unnecessary, as it covers the entire heart throughout the scan. This advance has multiplied system sensitivity. The first results (4) from CZT cameras have fuelled high expectations with regard to lower activity (5), shorter scan times (6), and possibly improved image quality (7). In particular, the multipinhole geometry may have the potential to decrease artifacts from extracardiac activity and from abdominal soft-tissue attenuation. This is of particular importance in obese subjects, who represent a growing proportion of patients undergoing evaluation for coronary artery disease and may benefit the most from multipinhole geometry. On the other hand, as a result of this geometry, the heart has to be centered in a much smaller field of view than with conventional cameras, and image acquisition may often be challenging in obese patients even after repositioning of the patient.

The aim of the present study was to systematically assess the feasibility of CZT MPI scanning in patients with high, very high, or excessive body mass index (BMI).

\section{MATERIALS AND METHODS}

\section{Study Population}

We enrolled 65 obese patients (BMI $\geq 30 \mathrm{~kg} / \mathrm{m}^{2}$ ) and 18 randomly selected nonobese patients (BMI $<30 \mathrm{~kg} / \mathrm{m}^{2}$ ) referred for the assessment of known or suspected coronary artery disease by 1-d stress/rest MPI on a CZT $\gamma$-camera (NM/CT 570c; GE Healthcare). Patients were assigned according to the World Health Organization into obese class I (BMI, 30-34.9 kg/m²), II (BMI, $\left.35-39.9 \mathrm{~kg} / \mathrm{m}^{2}\right)$, or III (BMI $\geq 40 \mathrm{~kg} / \mathrm{m}^{2}$ ). Class III patients were further categorized as "morbid obese" (BMI $\geq 40 \mathrm{~kg} / \mathrm{m}^{2}$ ) or "super obese" (BMI $\geq 45 \mathrm{~kg} / \mathrm{m}^{2}$ ) as previously suggested (8). We enrolled a minimum of 15 patients per group. In addition to BMI, the chest wall circumference of supine patients was measured at the level of the mammillae. The need to obtain written informed consent was waived because of the nature of the study, which included solely clinical data collection.

\section{Image Acquisition}

All patients underwent a 1-d pharmacologic stress/rest SPECT MPI protocol with standard adenosine infusion $(0.14 \mathrm{mg} / \mathrm{kg} / \mathrm{min}$ over $6 \mathrm{~min})$. After $392 \pm 47 \mathrm{MBq}$ of ${ }^{99 \mathrm{~m}} \mathrm{Tc}$-tetrofosmin had been 
TABLE 1

Patient Baseline Characteristics

\begin{tabular}{|c|c|}
\hline Characteristic $(n=81)$ & Value \\
\hline Mean age $\pm S D(y)$ & $63 \pm 11$ \\
\hline Men (\%) & 60 \\
\hline \multicolumn{2}{|l|}{$\mathrm{BMI} \pm \mathrm{SD}\left(\mathrm{kg} / \mathrm{m}^{2}\right)$} \\
\hline Overall & $38 \pm 9$ \\
\hline$\leq 29.9(n=18)$ & $25 \pm 2$ \\
\hline $30-34.9(n=17)$ & $33 \pm 1$ \\
\hline $35-39.9(n=15)$ & $37 \pm 1$ \\
\hline $40-44.9(n=16)$ & $42 \pm 2$ \\
\hline$\geq 45(n=15)$ & $50 \pm 6$ \\
\hline \multicolumn{2}{|l|}{ Cardiovascular risk factors (\%) } \\
\hline Hypertension & 71 \\
\hline Hypercholesterolemia & 61 \\
\hline Diabetes mellitus & 27 \\
\hline Smoking & 22 \\
\hline Positive family history & 29 \\
\hline \multicolumn{2}{|l|}{ Clinical history of coronary artery disease (\%) } \\
\hline Coronary artery disease & 35 \\
\hline Previous percutaneous coronary intervention & 29 \\
\hline Coronary artery bypass grafting & 11 \\
\hline Angina symptoms (CCS class $\geq 2$ ) & 26 \\
\hline Dyspnea (NYHA functional class $\geq \mathrm{II}$ ) & 22 \\
\hline \multicolumn{2}{|l|}{ Medication (\%) } \\
\hline Platelet aggregation inhibitors & 67 \\
\hline Antiischemic agents & 71 \\
\hline Lipid-lowering agents & 63 \\
\hline
\end{tabular}

administered, stress MPI images were acquired approximately $60 \mathrm{~min}$ later (during $5 \mathrm{~min}$ ) on the CZT camera. This acquisition was followed by rest MPI using the identical protocol several minutes after administration of a 3 times higher dose of ${ }^{99 \mathrm{~m}} \mathrm{Tc}-$ tetrofosmin $(1,143 \pm 133 \mathrm{MBq})$, as previously validated $(9,10)$. MPI scans were acquired using a multipinhole collimator and 19 stationary detectors simultaneously imaging 19 different views of the heart as previously reported (6). In brief, each detector contains $32 \times 32$ pixelated $(2.46 \times 2.46 \mathrm{~mm}) \mathrm{CZT}$ elements; the system design allows acquisition without detector or collimator motion, and a $10 \%$ symmetric energy window at $140 \mathrm{keV}$ was used. An unenhanced low-dose 64-slice CT scan with prospective triggering at $75 \%$ of the R-R interval was performed for attenuation correction (AC) of CZT MPI $(11,12)$.
Whenever the heart was out of the camera field of view (19-cm diameter) on supine CZT MPI scanning, the acquisition was immediately repeated on the same camera after repositioning the patient by inclination of the chest by $20^{\circ}-30^{\circ}$ or by shifting the body closer to the CZT detector. All 15 patients with a BMI $\geq 45$ $\mathrm{kg} / \mathrm{m}^{2}$ were additionally scanned on a conventional dual-head SPECT/CT camera (Infinia; GE Healthcare) at the same session according to our clinical routine for super obese patients. Briefly, emission data were acquired with a parallel-hole, low-energy, high-resolution collimator with a $20 \%$ symmetric window centered at $140 \mathrm{keV}\left(3^{\circ}\right.$ of rotation per stop, $180^{\circ}$ for each head, and $25 \mathrm{~s}$ per projection). After MPI, CT for AC was performed using the Infinia system at $140 \mathrm{keV}$ and $3.0 \mathrm{~mA}$ (13).

\section{MPI Image Reconstruction}

CZT images were reconstructed as previously described $(6,9)$ on a dedicated Xeleris workstation (GE Healthcare) applying an iterative reconstruction algorithm with maximum-likelihood expectation maximization. The software Myovation for Alcyone (GE Healthcare) was used for image reconstruction, and a Butterworth postprocessing filter was applied to the reconstructed slices. All MPI scans were reconstructed in standard axes (short axis, vertical long axis, and horizontal long axis), and polar maps of perfusion were generated using a commercially available software package (Cedars QGS/QPS; Cedars-Sinai Medical Center) (14).

\section{Image Quality Assessment}

MPI scans were visually scored for quality using an adapted 4-grade scoring system $(1=$ poor, $2=$ moderate, $3=$ good, and 4 = excellent) (15) by 2 masked experienced nuclear cardiologists based on predefined cardiac image characteristics (homogeneity of myocardial radionuclide uptake, integrity or truncation of left ventricle as previously defined (16), and position of left ventricle in the field of view) or interference from extracardiac radioactivity (no activity, minor activity adjacent to the myocardium but not affecting image interpretation, or excessive blood-pool activity or activity significantly reducing confidence in image interpretation). Discrepancies between the 2 readers were resolved by consensus. If the image quality was at least 3 or better, the MPI was considered diagnostic.

\section{Statistical Analysis}

Quantitative variables were expressed as mean $\pm \mathrm{SD}$, and categoric variables as frequencies or percentages. SPSS software, version 19.0 (IBM), was used for all statistical analysis. Receiveroperating-curve analysis was applied to measure the area under the curve (AUC) to determine optimal cutoffs for BMI and

TABLE 2

Image Quality in Patients with BMI $<45 \mathrm{~kg} / \mathrm{m}^{2}$

\begin{tabular}{|c|c|c|c|c|c|c|c|}
\hline \multirow[b]{2}{*}{ BMI $\left(\mathrm{kg} / \mathrm{m}^{2}\right)$} & \multirow[b]{2}{*}{$n$} & \multicolumn{2}{|c|}{ Without AC } & \multicolumn{2}{|c|}{ With AC } & \multirow[b]{2}{*}{$P$} & \multirow[b]{2}{*}{ Normals (\%) } \\
\hline & & Mean IQ & $\mathrm{IQ} \geq 3(n)$ & Mean IQ & $\mathrm{IQ} \geq 3(n)$ & & \\
\hline$<30$ & 18 & $3.6 \pm 0.5$ & 18 & $3.6 \pm 0.5$ & 18 & NS & 50 \\
\hline 30-34.9 & 17 & $3.4 \pm 0.6$ & 16 & $3.4 \pm 0.6$ & 16 & NS & 88 \\
\hline $35-39.5$ & 15 & $2.8 \pm 0.7$ & 10 & $2.9 \pm 0.5$ & 12 & NS & 67 \\
\hline $40-44.5$ & 16 & $1.9 \pm 0.8$ & 4 & $2.4 \pm 0.7$ & 8 & NS & 75 \\
\hline
\end{tabular}


TABLE 3

Image Quality in Patients with $\mathrm{BMI} \geq 45 \mathrm{~kg} / \mathrm{m}^{2}$

\begin{tabular}{|c|c|c|c|c|c|c|}
\hline \multirow[b]{2}{*}{ Type of MPI } & \multirow[b]{2}{*}{$n$} & \multicolumn{2}{|c|}{ Without AC } & \multicolumn{2}{|c|}{ With AC } & \multirow[b]{2}{*}{ Normals (\%) } \\
\hline & & Mean IQ & $\mathrm{IQ} \geq 3(n)$ & Mean IQ & $\mathrm{IQ} \geq 3(n)$ & \\
\hline \multicolumn{7}{|l|}{$\mathrm{CZT}$} \\
\hline Standard & 15 & $1.6 \pm 0.7$ & 2 & $2.1 \pm 0.8$ & 6 & 87 \\
\hline Repositioning & 8 & $2.8 \pm 1.0^{\star}$ & 5 & $3.1 \pm 0.8^{\star}$ & 6 & 75 \\
\hline SPECT & 15 & $3.4 \pm 0.5^{\star}$ & 15 & $3.6 \pm 0.5^{\star}$ & 15 & 87 \\
\hline $\begin{array}{l}{ }^{*} P<0.05 \text { vs. star } \\
\mathrm{IQ}=\text { image qualit }\end{array}$ & & & & & & \\
\hline
\end{tabular}

chest wall circumference to ensure diagnostic image quality. The Mann-Whitney U test and the Jonckheere Terpstra test were used to check for significant differences in image quality between the different modalities. $P$ values of less than 0.05 were considered statistically significant.

\section{RESULTS}

After exclusion of 2 patients with a BMI over $60 \mathrm{~kg} / \mathrm{m}^{2}$ from further analysis due to geometric inability to fit into either the CZT or the conventional SPECT camera, this study included 18 nonobese patients (BMI, $25 \pm 2 \mathrm{~kg} / \mathrm{m}^{2}$ ) and 63 obese patients. The patient baseline characteristics are given in Table 1. Eight patients were rescanned on the CZT camera after repositioning, as the heart was out of the field of view. The upper limit of chest circumference to fit into the gantry of the CZT (and the conventional SPECT) camera in the present study was $141 \mathrm{~cm}$. Chest circumference was closely related to BMI $(r=0.87, P<0.001)$. Image quality from the CZT scanner was significantly higher in men than in women, both with $\mathrm{AC}(3.1 \pm 0.1$ vs. $2.7 \pm 0.2, P<0.05)$ and without $\mathrm{AC}(2.9 \pm 0.1$ vs. $2.4 \pm$ 0.2 ). Heart size was comparable in nonobese and obese patients (end-diastolic volume, $92.3 \pm 42.9$ vs. $92.4 \pm$ $50.4, P=$ not statistically significant).

Image quality of the different BMI groups is given in Tables 2 and 3 . In patients with BMI $\geq 40 \mathrm{~kg} / \mathrm{m}^{2}, 81 \%$ of the CZT scans were nondiagnostic (Fig. 1), mainly because the cardiac position was out of focus, causing truncation artifacts of the left ventricle (Fig. 2). After application of $\mathrm{AC}$, this rate significantly decreased to $55 \%(P<0.05)$. In patients with $\mathrm{BMI} \geq 45 \mathrm{~kg} / \mathrm{m}^{2}$, the $\mathrm{CZT}$ image quality (no AC; $1.6 \pm 0.7$ AC, $2.1 \pm 0.8$ ) improved significantly (Fig. 3) after patient repositioning within the CZT camera (no AC, $2.8 \pm 0.7$ AC, $3.1 \pm 0.8 ; P<0.05$ ). Similarly, image quality from SPECT MPI was substantially and significantly higher (no AC, $3.4 \pm 0.5$; AC, $3.6 \pm 0.5 ; P<$ 0.001) than that from CZT MPI, reaching diagnostic image quality in every patient. Overall, $59(73 \%)$ of 81 scans were reported as normal.

Receiver-operating-curve analysis (Fig. 4A) revealed a cutoff BMI of $39 \mathrm{~kg} / \mathrm{m}^{2}$ to obtain diagnostic MPI quality for both non-AC images (AUC, 91\%; confidence interval,
0.85-0.97; $P<0.001$ ) and AC images (AUC, 84\%; confidence interval, $0.75-0.93 ; P<0.001)$. Similarly, for chest wall circumference, receiver-operating-curve analysis (Fig. 4B) revealed a cutoff of $123 \mathrm{~cm}$ to obtain diagnostic MPI quality for both non-AC images (AUC, 89\%; confidence interval, 0.81-0.96; $P<0.001$ ) and AC images (AUC, 81\%; confidence interval, 0.71-0.91; $P<0.001)$. Differences in image quality between non-AC and $\mathrm{AC}$ images were not statistically significant.

\section{DISCUSSION}

Our results suggest that for patients with morbid obesity, MPI on CZT cameras may often yield insufficient image quality; the patient's heart cannot get close enough to the detectors because the body mass is too high for the size of the gantry. As a result, in patients with $\mathrm{BMI} \geq 40 \mathrm{~kg} / \mathrm{m}^{2}$, $81 \%$ of non-AC scans and $55 \%$ of AC scans were nondiagnostic. By contrast, image quality was significantly better from a conventional $\gamma$-camera and was diagnostic in all patients.

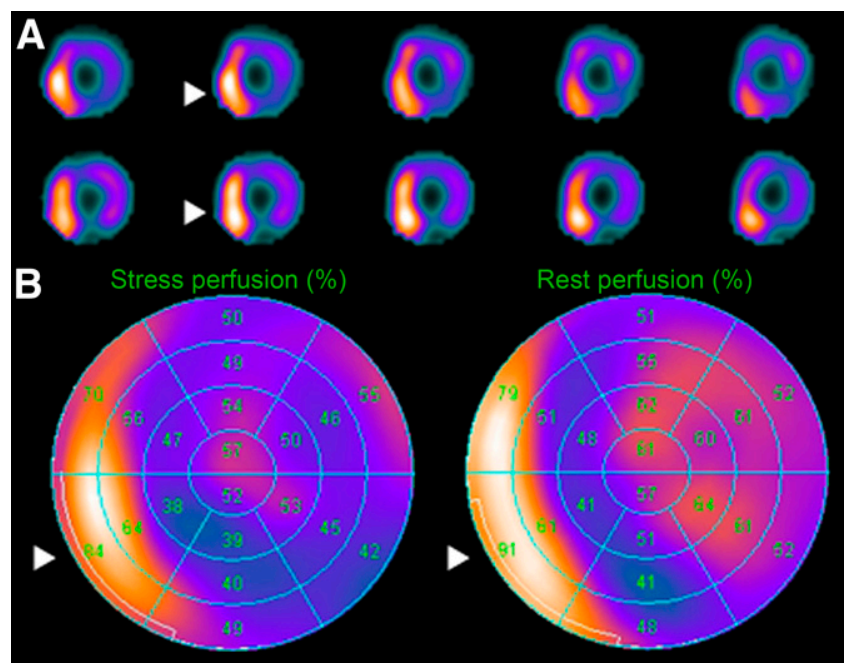

FIGURE 1. Obesity artifact in SPECT MPI. Typical artifact (banana shape; arrowheads) observed in CZT scanning of obese patients is shown on short-axis images (A) and corresponding polar maps (B). 


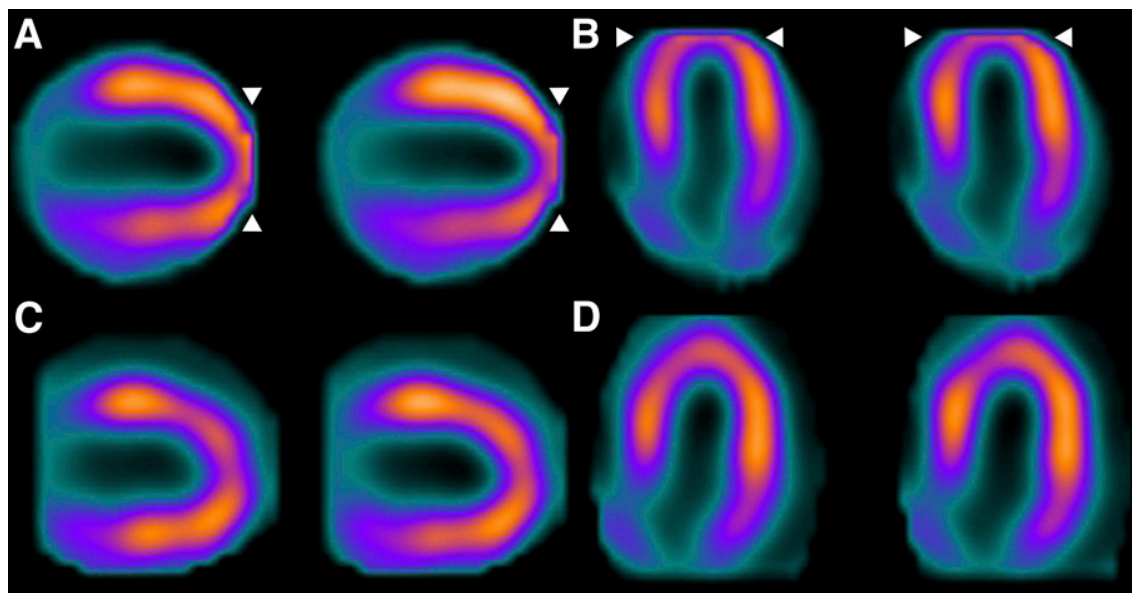

FIGURE 2. Apical truncation artifact. Eccentric position of heart causes apical truncation artifact (arrowheads), illustrated in vertical (A) and horizontal (B) long-axis views; for comparison, same views are given without truncation artifact (C and D).

Obesity may often affect the quality of SPECT MPI scans, mainly because of reduction of test specificity by diaphragm soft-tissue attenuation and extracardiac activity (17-21). CT-based AC has been found to compensate at least in part for inferior soft-tissue attenuation, resulting in improved SPECT MPI diagnostic (22) and prognostic (23) accuracy. The latest generation of $\gamma$-cameras with CZT detectors was expected to offer improved image quality in obese patients, as its detector geometry with pinhole collimation should help avoid image degradation by soft-tissue attenuation. Attenuation artifacts can nevertheless occur but can be corrected with x-ray-based CT attenuation maps (12). In the new generation of cardiac CZT cameras the target organ, the heart, has to be centered accurately within the field of view, which is substantially smaller than that on standard SPECT cameras. For most patients in our daily routine, this requirement does not present any limitation. However, in patients with morbid obesity this limitation becomes critical, particularly in a center such as ours, which receives regular referrals for preoperative MPI from an associated busy bariatric surgery program.

Our data show that AC may improve image quality but fails to solve the fundamental problem of imaging obese patients with CZT cameras. Even careful repositioning frequently remains unsuccessful in some very obese patients, in whom the standard SPECT camera remains the first-choice tool. By receiver-operating-curve analysis, we have identified a BMI of $39 \mathrm{~kg} / \mathrm{m}^{2}$ as the cutoff for direct scheduling of scanning on a standard camera. BMI rather than absolute weight is used to categorize obesity, as the absolute weight in tall persons may be high without reflecting obesity. Consequently, BMI rather than absolute weight is most appropriate for identifying patients who should not undergo CZT scanning. Very rarely, however, the patient's circumference exceeds the gantry even of such a camera, as was the case in 2 of our patients, rendering scanning impossible on any of our available devices. The ability of the patient to fit within the scanner should therefore be routinely tested before any tracer activity is injected.
It may be seen as a potential limitation of the present study that we did not assess the accuracy of CZT by comparing it with a reference standard such as invasive coronary angiography with fractional flow reserve. Such an assessment, however, was beyond the scope of the present feasibility study, which was designed to evaluate how severe obesity interacts with diagnostic image quality in the new-generation CZT $\gamma$-camera. Furthermore, not all patients were rescanned on a conventional SPECT camera for direct comparison, and therefore the relatively small size of the study subgroups may appear as a limitation. However, all patients with BMI $\geq 45 \mathrm{~kg} / \mathrm{m}^{2}(n=15)$ were rescanned, and this rescanning increased the rate of diagnostic image quality to $100 \%$. It may be reasonable to assume that in less obese patients, MPI on a standard SPECT camera would not be less successful. Similarly, only 8 of 15 patients-mainly those with nondiagnostic image quality after $\mathrm{AC}$-were repositioned and rescanned with the heart better centered in the field of view. However, the subjective discomfort induced by repositioning of

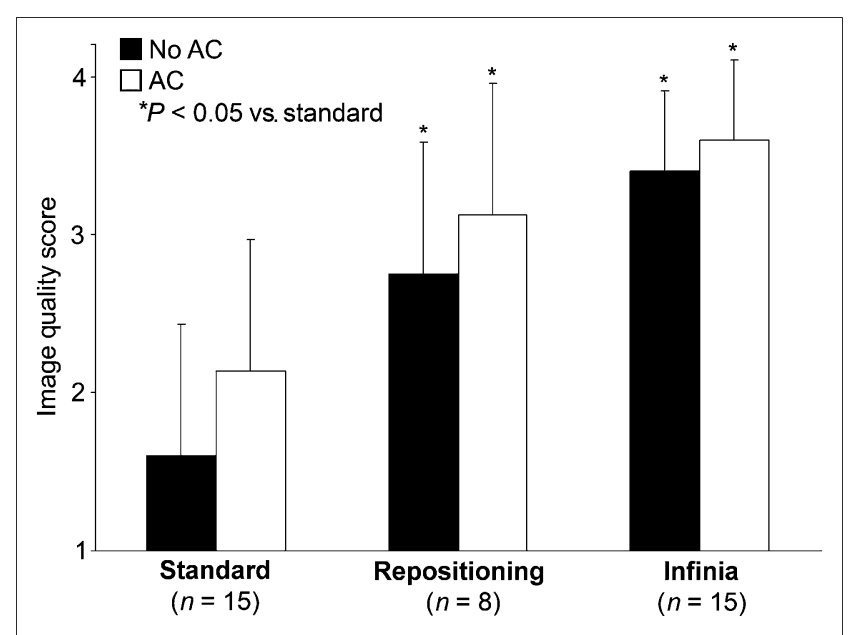

FIGURE 3. Image quality in patients with $B M I \geq 45 \mathrm{~kg} / \mathrm{m}^{2}$. Shown is image quality of standard CZT MPI $(n=15)$, CZT MPI after repositioning $(n=8)$, and conventional SPECT $(n=15)$. 

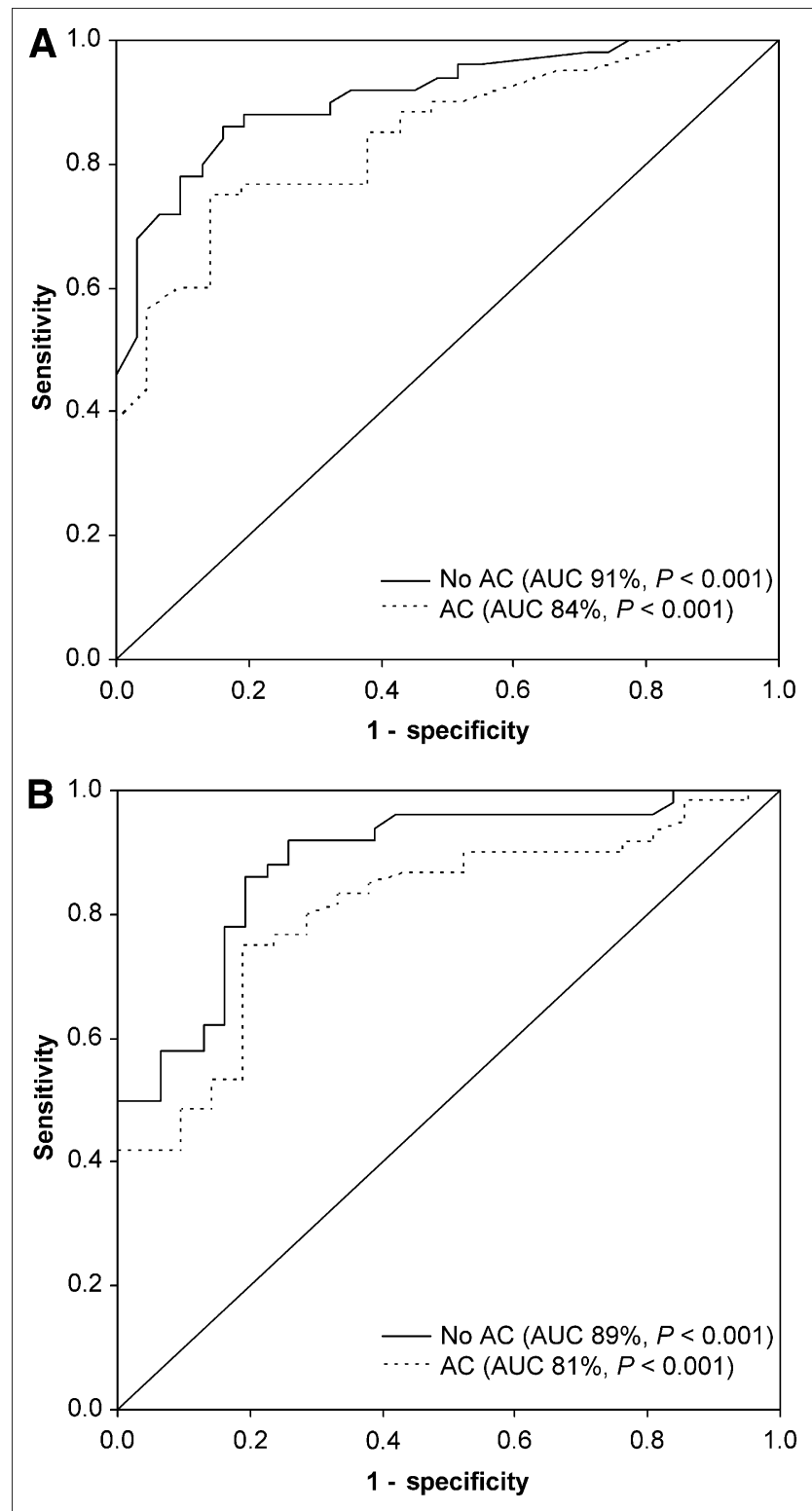

FIGURE 4. Receiver-operating-curve analysis for CZT MPI image quality and $\mathrm{BMI}(\mathrm{A})$ or chest wall circumference (B).

super obese patients was considered too high to justify such a maneuver in patients with diagnostic image quality. Although image quality was higher in men than women overall, the present study was not designed to provide such comparison data for each BMI group. Further, the present study used vasodilator stress although the use of physical exercise stress might have reduced extracardiac activity and potentially improved image quality. However, severe obesity often hampers the ability to perform adequate physical exercise. Finally, in this non-heart-failure population, heart size was similar in obese and nonobese patients. The role of increased heart size on image quality from CZT cameras with a small field of view remains to be evaluated in patients with an enlarged left ventricle.

\section{CONCLUSION}

The introduction of CZT $\gamma$-cameras, with their advantages over conventional SPECT cameras, has not solved the issue of image quality degradation from obesity. On the contrary, it appears that the actual design of CZT cameras, with pinhole collimation geometry and a smaller field of view, is more prone to artifacts in very obese patients. Therefore, patients with a BMI of $40 \mathrm{~kg} / \mathrm{m}^{2}$ and above should be scheduled on a conventional SPECT camera for MPI scanning.

\section{DISCLOSURE STATEMENT}

The costs of publication of this article were defrayed in part by the payment of page charges. Therefore, and solely to indicate this fact, this article is hereby marked "advertisement" in accordance with 18 USC section 1734 .

\section{ACKNOWLEDGMENTS}

We thank Ennio Mueller and Patrick von Schulthess for their excellent technical support. This study was supported by a grant from the Swiss National Science Foundation. No other potential conflict of interest relevant to this article was reported.

\section{REFERENCES}

1. Valenta I, Treyer V, Husmann L, et al. New reconstruction algorithm allows shortened acquisition time for myocardial perfusion SPECT. Eur J Nucl Med Mol Imaging. 2010;37:750-757.

2. Giorgetti A, Rossi M, Stanislao M, et al. Feasibility and diagnostic accuracy of a gated SPECT early-imaging protocol: a multicenter study of the Myoview Imaging Optimization Group. J Nucl Med. 2007;48:1670-1675.

3. Herzog BA, Husmann L, Buechel RR, et al. Rapid cardiac hybrid imaging with minimized radiation dose for accurate non-invasive assessment of ischemic coronary artery disease. Int J Cardiol. 2011;153:10-13.

4. Esteves FP, Raggi P, Folks RD, et al. Novel solid-state-detector dedicated cardiac camera for fast myocardial perfusion imaging: multicenter comparison with standard dual detector cameras. J Nucl Cardiol. 2009;16: 927-934.

5. Berman DS, Kang X, Tamarappoo B, et al. Stress thallium-201/rest technetium$99 \mathrm{~m}$ sequential dual isotope high-speed myocardial perfusion imaging. JACC Cardiovasc Imaging. 2009;2:273-282.

6. Herzog BA, Buechel RR, Katz R, et al. Nuclear myocardial perfusion imaging with a cadmium-zinc-telluride detector technique: optimized protocol for scan time reduction. J Nucl Med. 2010;51:46-51.

7. Sharir T, Ben-Haim S, Merzon K, Prochorov V, Dickman D, Berman DS. High-speed myocardial perfusion imaging initial clinical comparison with conventional dual detector anger camera imaging. JACC Cardiovasc Imaging. 2008; 1:156-163.

8. Sturm R. Increases in morbid obesity in the USA: 2000-2005. Public Health. 2007;121:492-496.

9. Buechel RR, Herzog BA, Husmann L, et al. Ultrafast nuclear myocardial perfusion imaging on a new gamma camera with semiconductor detector technique: first clinical validation. Eur J Nucl Med Mol Imaging. 2010; 37:773-778.

10. Fiechter M, Ghadri JR, Kuest SM, et al. Nuclear myocardial perfusion imaging with a novel cadmium-zinc-telluride detector SPECT/CT device: first validation versus invasive coronary angiography. Eur J Nucl Med Mol Imaging. 2011;38: 2025-2030.

11. Herzog BA, Buechel RR, Husmann L, et al. Validation of CT attenuation correction for high-speed myocardial perfusion imaging using a novel cadmiumzinc-telluride detector technique. J Nucl Med. 2010;51:1539-1544. 
12. Fiechter M, Ghadri JR, Wolfrum M, et al. Downstream resource utilization following hybrid cardiac imaging with an integrated cadmium-zinctelluride/64-slice CT device. Eur J Nucl Med Mol Imaging. 2012;39: 430-436.

13. Schepis T, Gaemperli O, Koepfli $\mathrm{P}$, et al. Use of coronary calcium score scans from stand-alone multislice computed tomography for attenuation correction of myocardial perfusion SPECT. Eur J Nucl Med Mol Imaging. 2007; 34:11-19.

14. Germano G, Kavanagh PB, Waechter P, et al. A new algorithm for the quantitation of myocardial perfusion SPECT. I: technical principles and reproducibility. J Nucl Med. 2000;41:712-719.

15. Askew JW, Miller TD, Ruter RL, et al. Early image acquisition using a solidstate cardiac camera for fast myocardial perfusion imaging. J Nucl Cardiol. 2011;18:840-846.

16. Wosnitzer B, Gadiraju R, Depuey G. The truncation artifact. J Nucl Cardiol. 2011;18:187-191.

17. Berman DS, Kang X, Nishina H, et al. Diagnostic accuracy of gated Tc-99m sestamibi stress myocardial perfusion SPECT with combined supine and prone acquisitions to detect coronary artery disease in obese and nonobese patients. J Nucl Cardiol. 2006;13:191-201.
18. Duvall WL, Croft LB, Corriel JS, et al. SPECT myocardial perfusion imaging in morbidly obese patients: image quality, hemodynamic response to pharmacologic stress, and diagnostic and prognostic value. J Nucl Cardiol. 2006; 13:202-209.

19. Gemignani AS, Muhlebach SG, Abbott BG, Roye GD, Harrington DT, Arrighi JA. Stress-only or stress/rest myocardial perfusion imaging in patients undergoing evaluation for bariatric surgery. J Nucl Cardiol. 2011; 18:886-892.

20. Elhendy A, Schinkel AF, van Domburg RT, et al. Prognostic stratification of obese patients by stress ${ }^{99 \mathrm{~m}} \mathrm{Tc}$-tetrofosmin myocardial perfusion imaging. $\mathrm{J} \mathrm{Nucl}$ Med. 2006;47:1302-1306.

21. Ghanem MA, Kazim NA, Elgazzar AH. Impact of obesity on nuclear medicine imaging. J Nucl Med Technol. 2011;39:40-50.

22. Masood Y, Liu YH, Depuey G, et al. Clinical validation of SPECT attenuation correction using $\mathrm{x}$-ray computed tomography-derived attenuation maps: multicenter clinical trial with angiographic correlation. J Nucl Cardiol. 2005; 12:676-686.

23. Pazhenkottil AP, Ghadri JR, Nkoulou RN, et al. Improved outcome prediction by SPECT myocardial perfusion imaging after CT attenuation correction. $\mathrm{J} \mathrm{Nucl}$ Med. 2011;52:196-200. 